\title{
Localization Effect in a 2D Superconducting Network without Disorder
}

\author{
B. Pannetier, C.C. Abilio, E. Serret, Th. Fournier and P. Butaud \\ CNRS-CRTBT, associé à l'Université Joseph Fourier \\ 25 Av. des Martyrs, 38042 Grenoble Cedex 9, France
}

J. Vidal

Groupe de Physique des Solides-CNRS, UMR 7588, Universités Paris 7 et Paris 6, 2 place Jussieu, 75251 Paris Cedex 05, France

\begin{abstract}
The superconducting properties of a two-dimensional superconducting wire network with a new geometry have been measured as a function of the external magnetic field. The extreme localization effect recently predicted for this periodic lattice is revealed as a suppression of the critical current when the applied magnetic field corresponds to half a flux quantum per unit cell. For this particular magnetic field, the observed vortex state configuration is highly disordered.
\end{abstract}

PACS[72.15, 73.23, 74.25]

\section{Introduction}

Superconducting wire networks are well known model systems which provide an unique experimental access [1] to the fascinating properties of the energy spectrum of tight binding electrons. For example the critical temperature of a superconducting network exhibits an oscillatory dependence with the magnetic field which very accurately describes the features of the ground state of the spectrum. The mapping of the superconducting transition line to the Landau Levels is a direct consequence of the analogy between the linear Ginzburg-Landau equation for the superconducting order parameter and the Schroedinger equation for noninteracting charged particles. Other superconducting properties such as the equilibrium magnetization [2] or the critical current [3] have also been shown to result directly from the Landau levels 
spectrum.

Recently a novel case of extreme localization induced by a magnetic field was predicted [4] for noninteracting electrons in a regular two dimensional lattice with the so-called T3 geometry (Fig.1, inset). This phenomenon is due to a subtle interplay between the lattice geometry and the magnetic field and occurs for a particular magnetic flux which corresponds to half a flux quantum per plaquette. For this special magnetic flux the hopping terms between neighbour sites interfere destructively and lead to a confinement of the electron motion within the so-called Aharonov-Bohm cages. The properties of these electronic states are associated with an interesting behaviour of the Landau level spectrum for the $T 3$ lattice at half frustration $f=1 / 2$. Here the frustration $f=\phi / \phi_{0}$ is defined as the magnetic flux per plaquette in units of the flux quantum $\phi_{0}=h / e$. Instead of forming energy bands, the highly degenerate eigenvalues merge into only 3 discrete levels. The confinement effect is a consequence of the non-dispersive character of the eigenstates. This contrasts with the case of a square lattice [5] where the eigenstates are dispersive and form broad energy bands $\epsilon(k, f)$ at every rational frustration.

The signature of the finite group velocity $v=\frac{1}{\hbar} \frac{\partial \epsilon}{\partial k}$ of such extended states for a superconducting system manifests itself in the ability of the superconducting wavefuntion to carry a supercurrent. A simple model based on the depairing current of a superconducting wire [6] was developped in [7,3] for superconducting wire networks. According to this model the critical current exhibits strong maxima at rational frustrations $f=p / q$ (p and q integer numbers) with strength proportional to the curvature of $\epsilon(k)$. The validity of this picture was confirmed by experiments in a square lattice [3]. An alternate formulation in terms of vortex pinning for commensurate vortex lattice at rational $f$ is fully equivalent.

The absence of dispersion $\epsilon(k)$ in the T3 lattice suggests that the corresponding superconducting network should be unable to carry a supercurrent at $f=1 / 2$.

\section{Experimental Results}

We have fabricated superconducting wire networks that reproduce the T3 geometry and we have studied the consequences of the above charge confinement phenomenon on the superconducting properties : critical temperature, critical current and vortex configuration.

The observed $T_{c}(H)$ transition line (Fig1.a) can be understood in its very details [8] from the ground state of the Landau level spectrum and will not be discussed here. The critical current curve measured at constant temperature, 


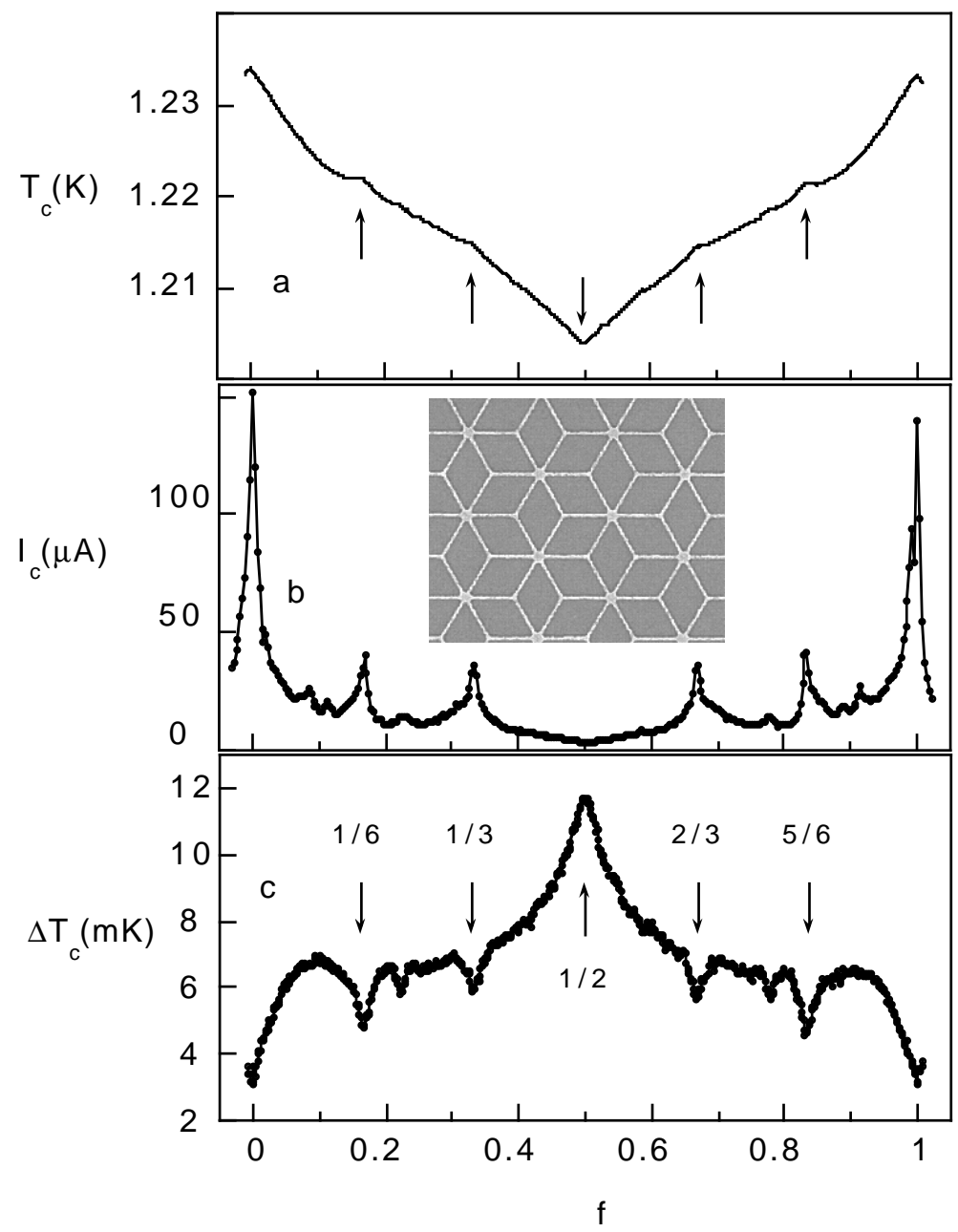

Fig. 1. Top : Critical temperature as a function of the frustration (frustration $f=1$ corresponds to an applied magnetic field of $B=0.234 \mathrm{mT}$ ). Middle : Critical current at fixed temperature $T=1.185 \mathrm{~K}$. Bottom : width of the superconducting transition. Note the behaviour at $f=1 / 2$ strongly differs from the behaviour at other rational frustrations $f=p / q$. Insert shows a micrograph of one part of the measured wire network : The aluminum strand are $1 \mu \mathrm{m}$ long with a cross section $100 \mathrm{x} 100 \mathrm{~nm}^{2}$.

$T=1.185 \mathrm{~K}$ is shown in Fig.1b. For each magnetic field the dynamic resistance characteristics was measured $v s$ increasing dc bias current. The critical current was defined as the threshold current for which the dynamic resistance exceeds $0.2 \%$ of the normal state resistance $\left(R_{N}\right)$. The width $\Delta T_{c}$ of the superconducting transition (Fig.1c) is defined as the difference between $T_{c}$ curves taken from criteria $0.6 R_{N}$ and $0.1 R_{N}$. The collection of data shown in Fig.1 reveals a quite unusual behaviour of the superconducting state of the T3 network. The usual picture that rational frustrations are characterized by (i) an upward cusp in the critical temperature curve, (ii) a sharp peak in the critical current and (iii) a dip in the transition width, is observed for values $0,1,1 / 6$, $1 / 3$, etc. . but is not observed for the simplest rational number $f=1 / 2$. Instead one observes a downward cusp in the critical temperature as in the case 
of an isolated single loop [10] while the critical current shows absolute minimum for this particular value. The suppression of critical current at $f=1 / 2$ reflects the effect of the band structure on the superfluid velocity and provides strong evidence of the non dispersive character of the eigenstates. The large broadening of the transition indicates that phase correlation between network sites cannot be established, leading to enhanced phase fluctuations.

This anomalous behaviour was never pointed out before. We claim that it is related to the localization effect discussed in ref[4]. Further experiments have been carried out recently in sample with wire strand lengthes as small as $0.5 \mu \mathrm{m}$ and will be published elsewhere [9].

The above discussed phenomena have their counterpart on the properties of the vortex lattice. The frustration $f$ is still the only control parameter which, here, can be viewed as the filling factor for vortices in the plaquettes of the lattice. We have used the Bitter decoration technique to visualize the vortex configuration in the $T 3$ lattice for $f=1 / 3$ and $f=1 / 2$. As shown recently [11] the decoration contrast can be significantly improved, in the case of superconducting arrays, by using the so-called flux compression method in which a thin superconducting bottom layer converts the network coreless vortices into well-defined Abrikosov vortices. Fig.2 compares the observed vortex patterns for $f=1 / 3$ and $f=1 / 2$. The $1 / 3$ case represents the reference situation where the natural triangular vortex lattice is commensurate with the underlying network. As expected we do observe an ordered state (Fig.2 left) which consists of a single domain state with a few point defects but without domain walls. The critical current peak and the pinning of the vortex lattice are two related phenomena whose origin is the dispersive curve $\epsilon(k)$. The nature of the vortex state at $f=1 / 3$ in the $T 3$ lattice is similar to that of the checkerboard commensurate state observed at half filling in a square lattice $[12,13]$. In contrast, the $f=1 / 2$ vortex structure does not exhibit any commensurate state (Fig. 2 right). A detailed analysis of the measured vortex correlation functions on an array containing several thousands plaquettes [9] shows no long range order under these conditions. Although there is no available theoretical model for the nature of the vortex ground state, the absence of a vortex commensurate state is likely consistent with the very high degeneracy of the electronic states in the tight-binding formulation. This result may have significant relevance on the more general field of frustrated systems. A detailed investigation of the ground states of vortices sitting on a Kagome lattice which is the dual of the T3 lattice is now in progress [14]. 


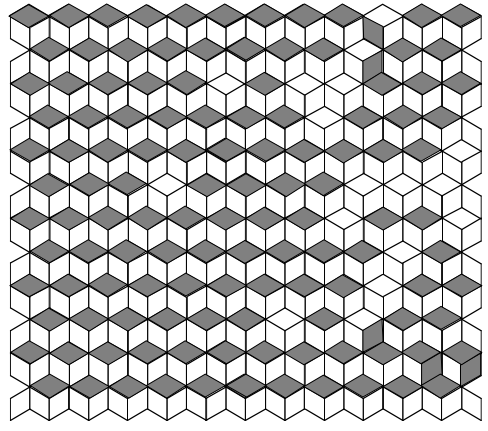

$f=1 / 3$

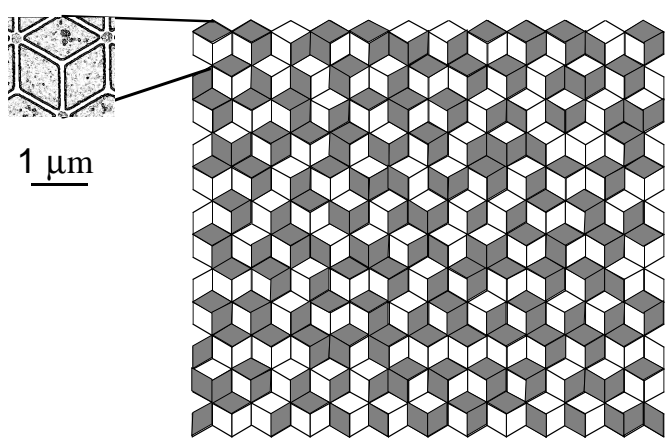

$f=1 / 2$

Fig. 2. The vortex configuration at $\mathrm{f}=1 / 3$ (left) and $\mathrm{f}=1 / 2$ (right). Inset : SEM picture showing three cells with one decorated vortex clearly visible in the middle of the upper cell. The samples for decoration are made of niobium epitaxially grown on a sapphire substrate. The elementary strands are $1 \mu \mathrm{m}$ long, $100 \mathrm{~nm}$ wide and $130 \mathrm{~nm}$ thick. The $70 \mathrm{~nm}$ thick uniform bottom layer allows for unambiguous identification of vortices inside the cells (inset)

\section{Conclusion}

It is noteworthy that the rationality of the frustration parameter at $f=1 / 2$ does not lead to a commensurate state in the T3 lattice. This uncommon situation is demonstrated by the decoration experiment. Actually the properties of the superconducting state at $f=1 / 2$ are reminiscent of the case of irrational frustration [15] in a square lattice. We believe that the anomalous superconducting properties presented in this paper are consistent with the localization effect predicted in ref [4]. The weakening of phase coherence by destructive interference is unambiguously observed. The mapping between the superconducting properties with the tight-binding problem allows for a formal connection between the current carrying superconducting states and the Landau level spectrum. The observed magnetic field dependence of the critical current can be accounted for qualitatively [8] by expressing the supercurrent in terms of the curvature of the band edge $\epsilon(k)$. However, this mapping is only valid in the vicinity of the critical temperature. The ordinary superconducting behaviour is recovered at lower temperature when the superconducting coherence length becomes smaller than the cell size. In this regime the large energy barriers prevents the vortex motion across the superconducting wires. A quite different situation may occur in Josephson junction arrays [16] where the superconducting phase fluctuations play a more important role. The absence of commensurate states in the $T 3$ lattice at $f=1 / 2$ may lead to interesting dynamics of the vortices at low temperatures. Apart from the superconducting systems, arrays of quantum wires [17] with the $T 3$ geometry are also expected to exhibit interesting properties related to this unique interference effect. 


\section{Acknowledgements}

We acknowledge B. Douçot, R. Mosseri and O. Buisson for fruitful discussions. The e-beam lithography and the SEM observations were carried out with PLATO organization teams and tools.

\section{References}

[1] B. Pannetier, J. Chaussy and R. Rammal, J. Phys. Lettres 44, L853 (1983); B. Pannetier, J. Chaussy, R. Rammal, Ph. Gandit and J.C. Villégier, Phys. Rev. Lett. 53, 1845 (1984).

[2] P. Gandit, J. Chaussy, A. Vareille and A. Tissier, EuroPhys. Lett. 3, 623 (1987).

[3] O. Buisson, M. Giroud and B. Pannetier, EuroPhys. Lett. 12, 727 (1990).

[4] J. Vidal, R. Mosseri and B. Douçot, Phys. Rev. Lett. 81, 5888 (1998).

[5] D.R. Hofstadter, Phys. Rev. B 14, 2239 (1976).

[6] M. Tinkham, Introduction to Superconductivity, MacGraw Hill Inc (1996).

[7] Y.Y. Wang, R.Rammal and B. Pannetier, J. Phys. 49, 2045 (1988).

[8] C.C. Abilio, P. Butaud, Th. Fournier, B. Pannetier, J. Vidal, S. Tedesco and B. Dalzotto, Phys. Rev. Lett. 83, 5102 (1999).

[9] E. Serret et al. to be published.

[10] W.A. Little and R. Parks, Phys. Rev. A 44, 97 (1964).

[11] A. Bezryadin, Y. Ovchinnikov and B. Pannetier, Phys. Rev. B 53, 8553 (1996).

[12] K. Runge and B. Pannetier, EuroPhys. Lett. 24, 737 (1993).

[13] H.D. Hallen, R. Seshadri, A.M. Chang, R.E. Miller, L.N. Pfeiffer, K.W. West, C.A. Murray and H.F. Hess, Phys. Rev. Lett. 71, 3007 (1993).

[14] P. Butaud et al. to be published.

[15] F. Yu, N. E. Israeloff, A.M. Goldman and R. Bojko, Phys. Rev. Lett. 68, 2535 (1992).

[16] see for example the recent review by P. Martinoli and Ch. Leemann, J. Low Temp. Phys. 118, 699 (2000).

[17] C. Naud and D. Mailly, Proceedings of the EPS conference, Montreux, march 2000. 